\title{
Differential drug effects on spontaneous and evoked pain behavior in a model of trigeminal neuropathic pain
}

This article was published in the following Dove Press journal: Journal of Pain Research

27 January 2017

Number of times this article has been viewed

\author{
K Deseure' \\ GH Hans ${ }^{2}$ \\ 'Department of Medicine, Laboratory \\ for Pain Research, University of \\ Antwerp, Wilrijk, ${ }^{2}$ Multidisciplinary \\ Pain Center, Antwerp University \\ Hospital, Edegem, Belgium
}

Purpose: Baclofen and morphine have shown efficacy against mechanical allodynia after infraorbital nerve chronic constriction injury (IoN-CCI). No drug effects have yet been reported on spontaneous trigeminal neuropathic pain. It has been proposed that the directed face grooming behavior that also develops following IoN-CCI offers a measure of spontaneous trigeminal neuropathic pain.

Subjects and methods: We examined the effects of a continuous 1-week infusion of $30 \mathrm{mg} /$ day carbamazepine (the first-line drug treatment for trigeminal neuralgia), $1.06 \mathrm{mg} /$ day baclofen, $4.18 \mathrm{mg}$ /day clomipramine, and $5 \mathrm{mg} /$ day morphine on spontaneous and mechanically evoked pain behavior (ie, directed face grooming and von Frey testing) in IoN-CCI rats.

Results: Isolated face grooming was significantly reduced in rats receiving carbamazepine and baclofen but not in clomipramine- or morphine-treated rats. All drugs showed significant antiallodynic effects; carbamazepine showed the strongest effects, whereas clomipramine had only minor efficacy.

Conclusion: The tested drugs have differential effects in the IoN-CCI model, and different neuropathological mechanisms may underlie the different somatosensory symptoms in this model. A mechanism-based approach may be needed to treat (trigeminal) neuropathic pain. The present data support IoN-CCI as a model of trigeminal neuralgia in which isolated face grooming is used as a measure of spontaneous neuropathic pain.

Keywords: trigeminal, infraorbital, neuropathic, allodynia, chronic, grooming

\section{Introduction}

Neuropathic pain remains a difficult condition to treat. The symptoms can be different between patients and in several cases who do not respond to treatment with traditional analgesics. ${ }^{1}$ A number of animal models are used to examine the responsible mechanisms in neuropathic pain and its pharmacological treatment. With regard to nociceptive pain, spontaneous manifestations of both the sensory and affective/motivational dimensions have been identified and validated. ${ }^{2-4}$ In contrast, animal studies of neuropathic pain have focused almost exclusively on evoked nocifensive behaviors that reflect hypersensitivity to mechanical and thermal stimulations (eg, vocalization and withdrawal responses). Unfortunately, neuropathic pain patients mostly suffer from spontaneous pain, and not from hypersensitivity. Furthermore, it has been argued that the pathophysiology of acutely evoked responses and hypersensitivity is different from that of spontaneous chronic pain. ${ }^{3,5,6}$ Therefore, an important aspect of translational pain research should be the identification and measurement of spontaneous pain in animals that are not being probed with stimulations that are superimposed on the basal condition.
Correspondence: K Deseure Department of Medicine, Laboratory for Pain Research, University of Antwerp, Universiteitsplein I, 2610 Wilrijk, Belgium Tel +32 32652561

Email kristof.deseure@uantwerp.be 
Unilateral chronic constriction injury (CCI) of the rat's infraorbital nerve (IoN) has been demonstrated to increase nocifensive behaviors in response to mechanical stimulation of the IoN territory and, importantly, to induce episodes of spontaneous face grooming directed to the territory of the injured IoN. ${ }^{7-9}$ The experiments presented here further assess the validity of isolated face grooming as an expression of spontaneous neuropathic pain and examine the effects of the established analgesics carbamazepine, baclofen, clomipramine, and morphine on this behavior.

Carbamazepine, a neuronal voltage-gated sodium channel blocker, is the first-line treatment of trigeminal neuralgia. Although some patients may not tolerate or become refractory to carbamazepine, many experience significant pain relief. ${ }^{10-12}$ In the IoN-CCI model, carbamazepine was found to be effective against mechanical allodynia only at doses that also induced sedation. ${ }^{13}$ The GABA-B receptor agonist, baclofen, has also been proven to be useful in treating trigeminal neuralgia. ${ }^{14-16}$ In the IoN-CCI model, both acute and continuous administration of baclofen significantly reduced mechanical allodynia. ${ }^{8,17,18}$ Tricyclic antidepressants (TCAs) are widely used against neuropathic pain. However, the TCA clomipramine was found to be devoid of antiallodynic efficacy after IoN-CCI. ${ }^{13}$ Finally, both acute and continuous morphine administration has proven effective against hyperresponsiveness to mechanical stimulation in the IoN-CCI model..$^{8,17,18}$ Here, we used nonsedative, continuous dose regimens to determine the effects of carbamazepine, baclofen, clomipramine, and morphine on both manifestations of spontaneous neuropathic pain (ie, face grooming) and mechanical allodynia.

\section{Subjects and methods Subjects}

Male Sprague-Dawley rats ( $\mathrm{n}=72$, weight $200-225 \mathrm{~g}$ on arrival, ie, 7 weeks old; Charles River, L'Arbresle cedex, France) were used. The rats were housed in solid-bottom polycarbonate cages in a colony room $\left(21 \pm 2^{\circ} \mathrm{C}\right.$; relative humidity $[\mathrm{RH}]=40 \pm 10 \%$ ). Water and food were available ad libitum. The rats were kept under a reversed 12:12 h dark/ light cycle (lights on at $7 \mathrm{pm}$ ). Animals were treated and cared for according to the guidelines of the Committee for Research and Ethical Issues of the International Association for the Study of Pain (IASP). ${ }^{19}$ The protocol was approved and performed in accordance with the Institutional Ethics Committee guidelines for animal research (Ethische Commissie Dierproeven, Universiteit Antwerpen).

The rats were allowed to acclimate for 16 days to the housing conditions before surgery, and they were habituated to the test procedure 7,6, and 4 days before preoperative baseline data were obtained. Habituation and testing were conducted in a darkened room (light provided by a $60 \mathrm{~W}$ red light bulb suspended $1 \mathrm{~m}$ above the observation area) with 45 $\mathrm{dB}$ background noise. The rats were individually transported from the colony to the test room in a covered plastic cage without bedding $(l \times w \times h, 24 \mathrm{~cm} \times 14 \mathrm{~cm} \times 17 \mathrm{~cm})$.

\section{Study design}

Baseline data were obtained 2 days before surgery and on postoperative days +5 (face grooming behavior) and $+4,+13$, and +25 (von Frey testing).

Rats were randomly assigned to one of six experimental drug groups ( $\mathrm{n}=12 /$ group, $\mathrm{cf}$ infra) and implanted with an osmotic pump after behavioral testing on postoperative day +5 , ie, at a time at which face grooming behavior is most strongly increased. ${ }^{7}$ Face grooming behavior was observed on postoperative days $+6,+8,+11$, and +26 . On postoperative day +11 , osmotic pumps were removed after the observation of face grooming behavior.

Rats were rerandomized and assigned to one of the six experimental drug groups and implanted with an osmotic pump for a second time on postoperative day +26 , ie, at a time at which mechanical allodynia had fully developed. ${ }^{7}$ von Frey testing was performed on postoperative days +27 , +29 , and +33 . On postoperative day +33 , osmotic pumps were removed after von Frey testing.

\section{Surgery}

Unilateral ligation of the IoN was performed as described elsewhere. ${ }^{7}$ The rats were anesthetized with pentobarbital $(60 \mathrm{mg} / \mathrm{kg}$, ip) and were treated with atropine $(0.1 \mathrm{mg} / \mathrm{kg}$, ip). All surgeries were performed under direct visual control using a Zeiss operation microscope $(\times 10-25)$. The head of the rat was fixed in a stereotaxic frame, and a mid-line scalp incision was made, exposing the skull and nasal bone. The infraorbital part of the IoN was exposed using a surgical procedure similar to those described elsewhere. ${ }^{20,21}$ The edge of the orbit, which is formed by the maxillary, frontal, lacrimal, and zygomatic bones, was dissected free. To enable access to the IoN, the orbital contents were gently deflected with a cotton-tipped wooden rod. The IoN was dissected free at its most rostral extent in the orbital cavity, just caudal to the infraorbital foramen. Two chromic catgut ligatures (5-0) were loosely tied around the IoN ( $2 \mathrm{~mm}$ apart). To obtain the desired degree of constriction, a criterion proposed by Bennet and $\mathrm{Xie}^{22}$ was applied: the ligatures reduced the diameter of the nerve by a just noticeable amount and retarded but did not 
interrupt the circulation through the superficial vasculature. The scalp incision was closed using polyester sutures (4-0). In the sham-operated rats, the IoN was exposed on one side using the same procedure, but the exposed IoN was not ligated.

Alzet osmotic pumps (model 2ML1; nominal pump rate, $10 \mu \mathrm{L} / \mathrm{h}$; Charles River) were implanted under anesthesia. Rats were placed in a cage under $4 \%$ isoflurane. When the rats were fully anesthetized (after ca 3-4 min), they were taken out of the cage and shaved and placed under a mask with $2.5 \%$ isoflurane. The shaved dorsal area was disinfected with Hibitane $^{\circledR}\left(0.5 \%\right.$ chlorhexidine in $70^{\circ}$ alcohol $)$. An incision of $\sim 2 \mathrm{~cm}$ was made in the skin. A subcutaneous pocket was created with hemostatic forceps, and $0.1 \mathrm{~mL}$ of an antibiotic (Pentrexyl ${ }^{\circledR}$, Na ampicillin, $500 \mathrm{mg}$ in $5 \mathrm{~mL}$ saline; S.A. Bristol-Myers Squibb, Brussels, Belgium) was released into the pocket with a sterile syringe $(1 \mathrm{~mL}$ Luer). The pump was inserted subcutaneously with the opening toward the rat's head. The incision was closed with four stainless steel staples (Slim Body Skin Stapler, Appose ULC auto suture 35; Covidien Mansfield, MA, USA) and sprayed with Aluspray ${ }^{\circledR}$.

Removal or replacement of the osmotic pumps was performed under isoflurane anesthesia as described earlier. The staples were removed from the skin using a hemostatic forceps, and an incision was made $\sim 1 \mathrm{~cm}$ from the previous incision.

\section{Observation of face grooming behavior}

Testing consisted of the observation of free behavior. The rats were observed in a transparent plastic cage with a mirrored back $(l \times w \times h, 24 \mathrm{~cm} \times 14 \mathrm{~cm} \times 17 \mathrm{~cm})$. Behavior was videotaped for $10 \mathrm{~min}$, and an experimenter who was blind to the experimental group to which the rat belonged analyzed the data.

The amount of time spent on face grooming (ie, movement patterns in which paws contact facial areas) ${ }^{7}$ was determined. A distinction was made between isolated face grooming and face grooming during body grooming. ${ }^{17}$ If a sequence was neither preceded by nor followed by body grooming (ie, movement patterns in which the paws, tongue, and incisors are brought into contact with a body area other than the face or the forepaws), ${ }^{7}$ the episode was categorized as isolated face grooming. If body grooming was present before or after a sequence of face grooming actions, the episode was categorized as face grooming during body grooming.

\section{Mechanical stimulation testing}

A graded series of five von Frey hairs (Pressure Aesthesiometer $^{\mathbb{B}}$; Stoelting Co, Chicago, IL, USA) were used. The force required to bend the filaments was $0.02,0.16,0.40,1.00$,and
$2.00 \mathrm{~g}$, respectively. The stimuli were applied within the IoN territory, near the center of the vibrissal pad, on the hairy skin surrounding the mystacial vibrissae. Within each animal, stimuli were applied in an ascending order of intensity. After a stimulus intensity was applied to one side, it was applied to the other side before moving on to the next stimulus intensity. The order in which the ipsilateral and contralateral sides were stimulated was randomized. The scoring system described by Vos et $\mathrm{al}^{7}$ was used to evaluate the response of the rats to the stimulation. The response was observed to belong to one of the following response categories: (score 0 ) no response; (score 1 ) detection $=$ the rat turns the head toward the stimulating object, and the stimulus object is then explored; (score 2) withdrawal reaction $=$ the rat turns the head slowly away or pulls it briskly backward when the stimulation is applied, and sometimes, a single face wipe ipsilateral to the stimulated area occurs; (score 3 ) escape/attack = the rat avoids further contact with the stimulus object, either passively by moving its body away from the stimulating object to assume a crouching position against the cage wall or actively by attacking the stimulus object, making biting and grabbing movements; and (score 4) asymmetric face grooming $=$ the rat displays an uninterrupted series of at least three face-wash strokes directed toward the stimulated facial area. For each rat, and at every designated time, a mean score for the five von Frey hairs was determined.

\section{Drugs and doses}

Carbamazepine (30 mg/day), baclofen (1.06 mg/day), morphine hydrochloride ( $5 \mathrm{mg}$ /day), and clomipramine $(4.18 \mathrm{mg} /$ day $)$ were used. The drugs were dissolved in vehicle (= dimethyl sulfoxide, propylene glycol, ethyl alcohol, and acetone at a ratio of 42:42:15:1) ${ }^{23}$ and were subcutaneously administered with one osmotic pump. The sham-operated and IoN-CCI vehicle control rats were implanted with a vehicle pump. The doses refer to the free base and were chosen based on previous studies (efficacy and side effects) and taking into account solubility limitations. . $^{13,18,23-25}$

\section{Statistical analysis}

Data are expressed as mean \pm standard error of the mean (SEM) and were analyzed (IBM SPSS Statistics 22 software) using a repeated measures analysis of variance (ANOVA) with observations (ie, rats were tested on different time points) as the within-subjects factor and treatment as the between-subjects factor. This analysis was followed by a unifactorial ANOVA per time point and post hoc comparisons using Dunnett's pairwise multiple comparisons with IoN-CCI vehicle rats as the control group. 


\section{Results}

\section{Face grooming}

The amount of time spent on isolated face grooming behavior in IoN-ligated animals was significantly different from that in sham-operated animals (groups $\times$ time interaction: $F[4,88]=9.77, P<0.001$ ) (Figure 1). Unifactorial ANOVA per time point, with experimental groups as betweensubjects factor, showed significant differences between the experimental groups on all postoperative time points $(F[5,69] \geq 4.36, P \leq 0.041)$. As indicated with asterisks in Figure 1, post hoc comparisons between the different drug groups, on the one hand, and IoN-ligated control rats, on the other hand, showed significant drug effects for carbamazepine and baclofen on all time points, but not for morphine or clomipramine.
The amount of time spent on face grooming during body grooming in IoN-ligated animals was not significantly different from that in sham-operated animals (groups $\times$ time interaction: $F[4,84]=1.41$, not significant $[\mathrm{NS}])$.

\section{Mechanical stimulation}

Response scores to ipsilateral mechanical stimulation in IoN-ligated animals were significantly different from those in sham-operated animals (groups $\times$ time interaction: $F[4,88]=18.48, P<0.001$ ) (Figure 2). On postoperative day +25 , no significant differences were found between the drug groups based on the first $(F[4,57]=1.68, \mathrm{NS})$ or the second $(F[4,57]=0.63, \mathrm{NS})$ randomization. Unifactorial ANOVA per time point, with experimental groups as between-subjects factor, showed significant differences between the experimental
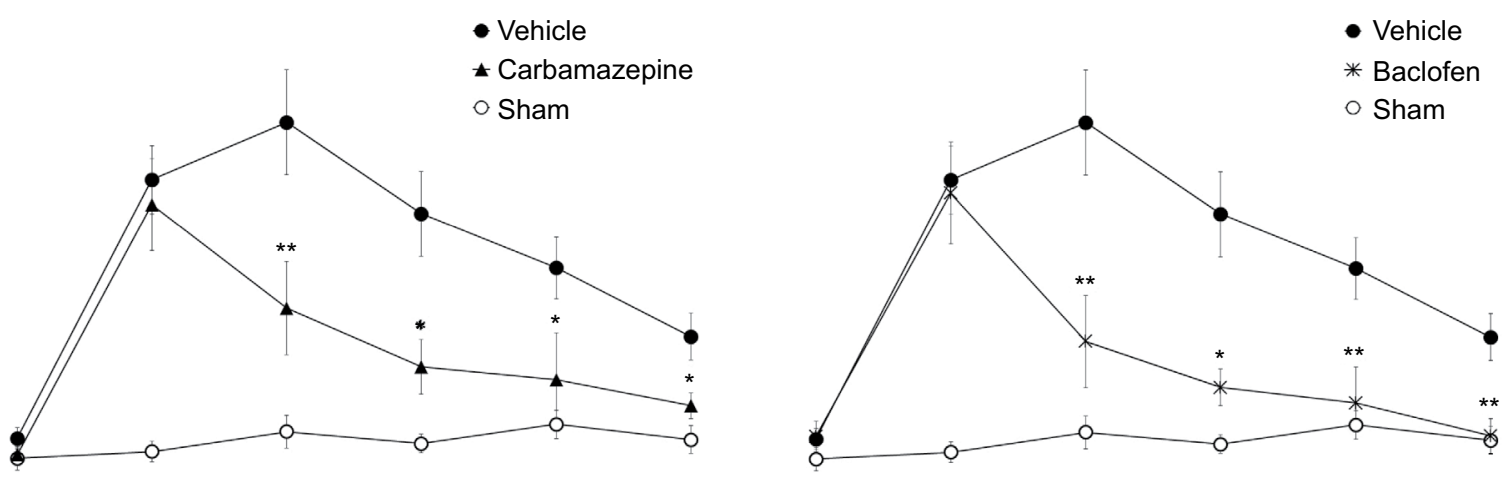

C
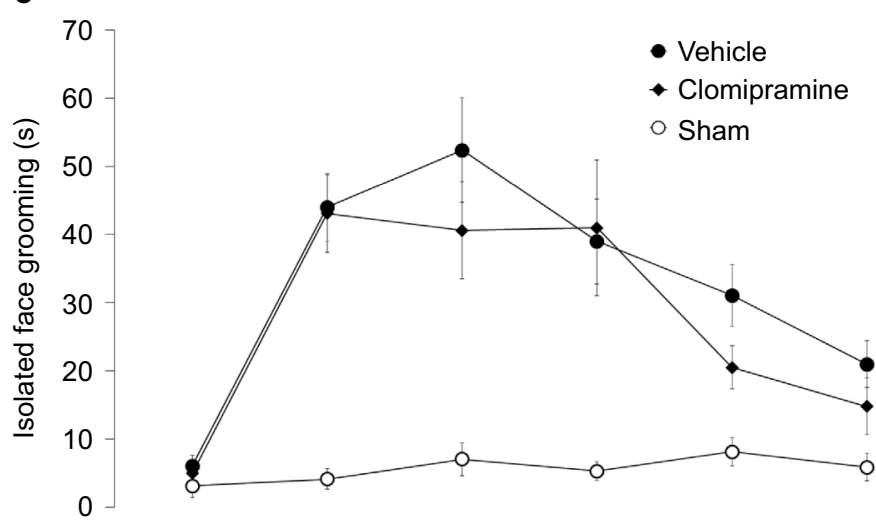

D
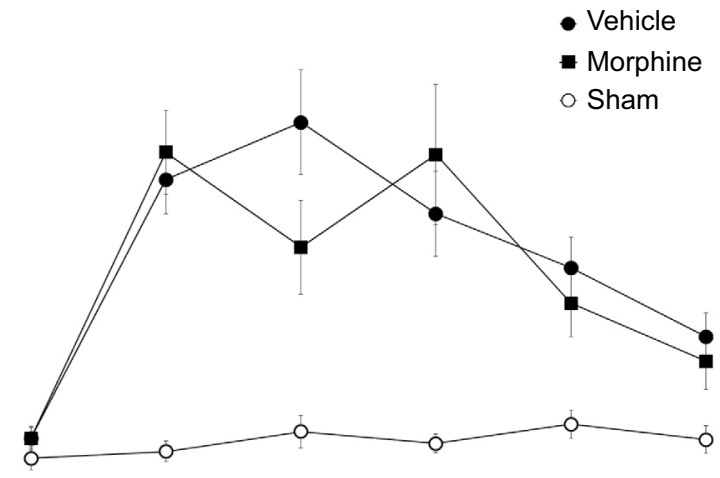

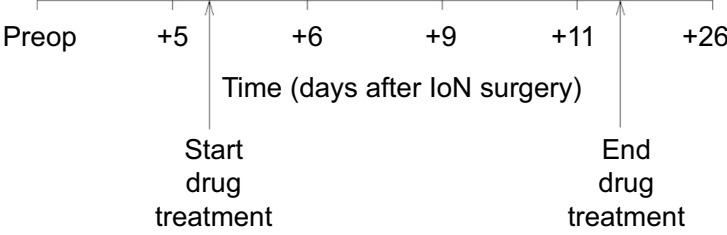

Figure I The effects of carbamazepine (A), baclofen (B), clomipramine (C), and morphine (D) on isolated face grooming behavior in loN-CCl-operated rats.

Notes: Data points represent the mean ( \pm SEM; $n=12$ per group) amount of isolated face grooming before (preop) and 5-26 days after loN surgery. Drug or vehicle infusion via osmotic pumps started immediately after behavioral testing on postoperative day +5 ; osmotic pumps were removed immediately after behavioral testing on postoperative day + I I. Asterisks indicate a significant difference compared to the vehicle control rats $(* P<0.05 ; * * P<0.0$ I).

Abbreviation: loN-CCl, infraorbital nerve chronic constriction injury 


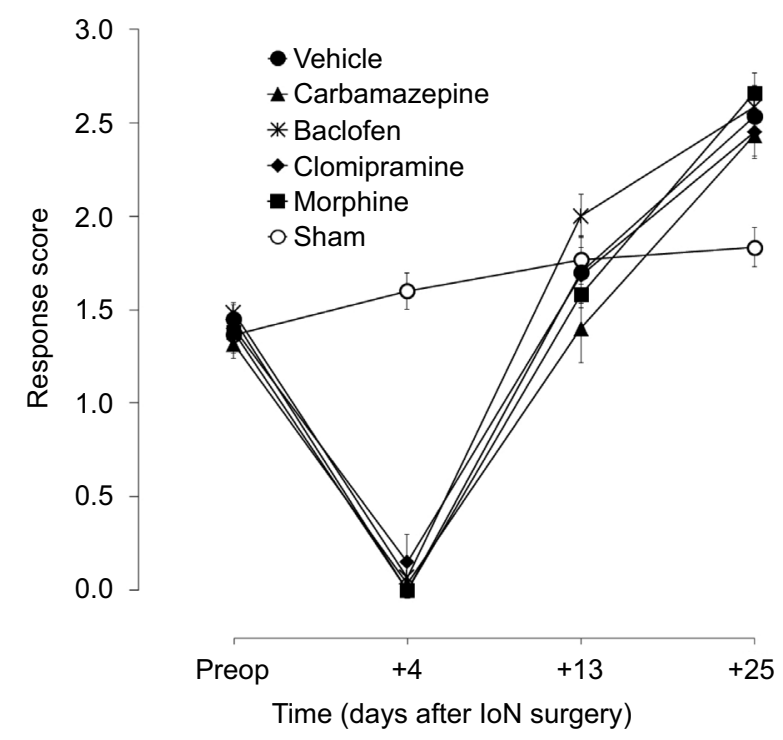

Figure 2 Time course of the effects of loN-CCl and sham surgery on responsiveness to mechanical stimulation.

Notes: Data points represent the mean ( \pm SEM; $n=12$ per group) response score to von Frey hair stimulation of the ipsilateral territory of the ligated nerve before (preop) and 4-25 days after loN surgery. No drugs were administered on the selected time points. Abbreviation: loN-CCl, infraorbital nerve chronic constriction injury.

groups on all postoperative time points $(F[5,69] \geq 6.59$, $P<0.001)$ except day $+13(F[5,69]=1.59$, NS $)$. As indicated with asterisks in Figure 3, post hoc comparisons between the different drug groups, on the one hand, and IoN-ligated control rats, on the other hand, showed significant drug effects for carbamazepine, baclofen, and morphine on all time points and for clomipramine on postimplantation days +3 and +7 , but not on postimplantation day +1 .

Response scores to contralateral mechanical stimulation in IoN-ligated animals were not significantly different from those in sham-operated animals (groups $\times$ time interaction: $F[4,88]=1.65$, NS).

\section{Discussion}

Confirming the results from previous studies, IoN ligation caused a significant increase in isolated face grooming that peaked during the first week after surgery ${ }^{7,9}$ and a biphasic development of mechanical allodynia with hyperresponsiveness to mechanical stimulation occurring several weeks after initial hyporesponsiveness during the first postoperative week. ${ }^{7,18,25}$ Carbamazepine reduced both isolated face grooming and mechanical hyperresponsiveness. The use of carbamazepine has been particularly well established for treating trigeminal neuralgia, although it may also have a beneficial effect on painful diabetic neuropathy. ${ }^{10,26-28}$ It is an anticonvulsant drug that exerts membrane-stabilizing properties by nonspecifically blocking sodium channels ${ }^{29}$ and reduces neuronal excitability in sensitized C-fibers. It has been shown that carbamazepine inhibits spontaneous and electrically evoked high-frequency firing in peripheral nerve neuromas. ${ }^{30}$ Voltage-gated sodium channels play an important role in the spontaneous firing and ectopic excitability in injured sensory nerves. ${ }^{31,32}$ Ectopic and spontaneous firing has been identified as one of the (peripheral) mechanisms contributing to the development of neuropathic pain. ${ }^{33-35}$ Thus, it can be argued that carbamazepine produces its analgesic effect by reducing spontaneous firing after peripheral nerve injury. Previous animal studies have shown that carbamazepine produces significant effects against mechanical allodynia after both IoN ligation ${ }^{13}$ and sciatic nerve ligation ${ }^{36}$ and protects against the trigeminal pain induced by applying bradykinin onto the rat's tooth pulp. ${ }^{37}$ However, in these studies, the effective doses also induced sedation. In this study, carbamazepine showed no overt behavioral side effects and selectively reduced the isolated face grooming behavior without significantly affecting face grooming during body grooming. The behavioral selectivity of this effect aligns with the rotarod test data in a previous study ${ }^{13}$ and confirms the usefulness of face grooming during body grooming as a control measure for nonspecific effects on isolated face grooming. ${ }^{8,17}$ It remains unclear why, in this study, carbamazepine significantly reduced the isolated face grooming and allodynia-like behavior but failed to produce a significant effect in a previous study against allodynia-like behavior using the same model and similar treatment conditions. ${ }^{13}$ Rats received the same dose of carbamazepine (expressed as milligram per kilogram per hour), but in this study, administration was done continuously via a subcutaneously implanted pump as opposed to repetitive intraperitoneal (ip) injections. In this study, rats were tested for the first time $24 \mathrm{~h}$ after the start of drug treatment as opposed to following five repetitive injections (at 72 min intervals). The longer treatment duration may at least be partially responsible for the difference in efficacy. Furthermore, it must be noted that in this study, responses to mechanical stimulation were quantified using the graded scoring system described by Vos et al, ${ }^{7}$ whereas Idänpään-Heikkilä and Guilbaud ${ }^{13}$ used a threshold paradigm to quantify the responses. Finally, stress induced by repetitive ip injections may have affected the behavioral responses of the animals to mechanical stimulation.

Baclofen has a documented analgesic efficacy against trigeminal neuralgia. ${ }^{14-16}$ Administered under the same ${ }^{18}$ and similar ${ }^{13}$ conditions as in this study, it significantly reduced hyperresponsiveness to mechanical stimulation in the IoNCCI model. It has now been shown that baclofen also counteracts spontaneous trigeminal neuropathic pain. The present 
A

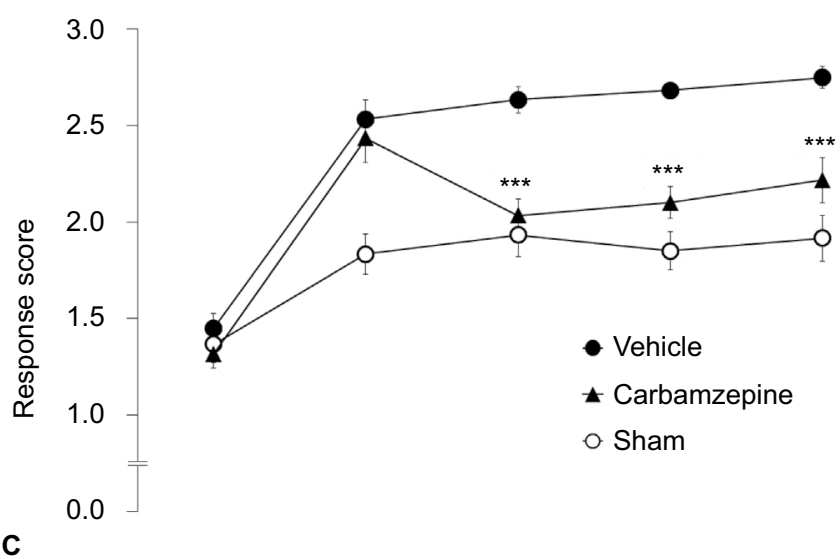

B

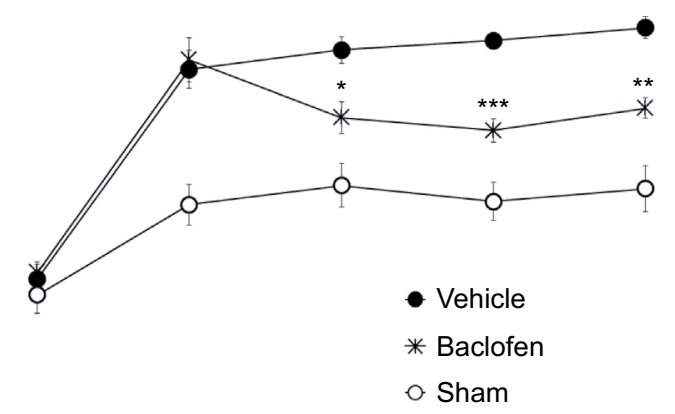

D
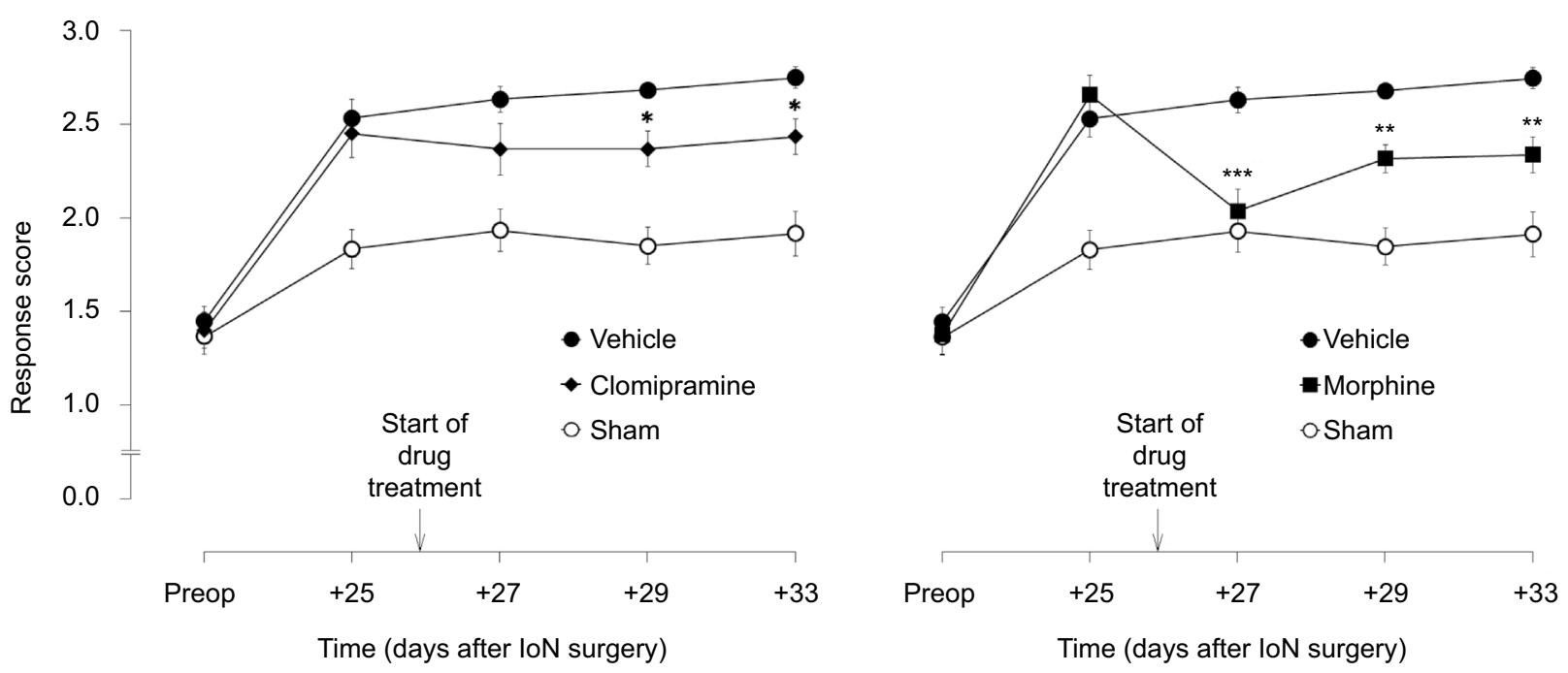

Figure 3 The effects of carbamazepine (A), baclofen (B), clomipramine (C), and morphine (D)

Notes: Data points represent the mean $( \pm$ SEM; $n=12$ per group) response score to von Frey hair stimulation of the ipsilateral territory of the ligated nerve before (preop) and 25-33 days after loN surgery. Drug or vehicle infusion via osmotic pumps started on postoperative day +26 ; osmotic pumps were removed after behavioral testing on postoperative day +33 . Asterisks indicate a significant difference compared to the vehicle control rats $(* P<0.05 ; * * P<0.01 ; * * * P<0.00 \mathrm{I})$.

Abbreviation: loN-CCl, infraorbital nerve chronic constriction injury.

results thus confirm previous findings and strengthen the clinical use of baclofen as a second-line drug for the treatment of trigeminal neuralgia.

For years, it was believed that morphine was ineffective in treating neuropathic pain. ${ }^{38}$ However, more recent studies have demonstrated the efficacy of morphine against neuropathic pain in both animals and humans. ${ }^{36,39-41}$ Studies from our own laboratory have also demonstrated antiallodynic effects in morphine-treated animals in the IoNCCI model of trigeminal neuropathic pain..$^{18,42,43}$ However, when infused continuously ( $5 \mathrm{mg} /$ day), this antiallodynic efficacy decreased over time; therefore, by the end of the 2-week treatment period, no significant antiallodynic effect could be observed. In this study, no significant decrease was observed in the amount of isolated face grooming in the animals treated with morphine. It must be considered that the rapid development of tolerance ${ }^{18}$ may have made it impossible to detect any action of morphine on isolated face grooming. It is also clinically well known that morphine may induce or intensify itching. ${ }^{44,45}$ It could be argued that, in the current model, opioid-induced pruritus may increase grooming behavior and thus counteract the analgesic effects of morphine on isolated face grooming. However, using different pruritogens and algogens, it has been shown that a clear distinction can be made between itch-evoked scratching (of the hindlimbs) and pain-evoked wiping (of the forelimbs). ${ }^{46,47}$ Furthermore, no increase in face grooming during body grooming was observed in morphine-treated animals. Still, a small increase in face wiping has been reported after intracisternal administration of morphine. ${ }^{45}$ Therefore, it cannot be totally excluded that morphine-induced pruritus counteracted (ie, by increasing face grooming) a potential analgesic effect (ie, decrease) on the isolated face grooming. 
A small decrease in mechanical hyperresponsiveness was found in clomipramine-treated animals that became significant 3 days after the start of drug treatment. No effect on isolated face grooming was found. As with carbamazepine, it is unclear why clomipramine failed to produce a significant antiallodynic effect in a previous study using the same IoN-CCI model and similar treatment conditions. ${ }^{13}$ As with carbamazepine, the same comments on the route and duration of administration apply to clomipramine (cf supra). It was also shown that the same dose of clomipramine effectively reduced mechanical hyperalgesia after loose ligation of the sciatic nerve. ${ }^{48}$ It should also be noted that a higher dose of clomipramine was reported to reduce mechanical hyperalgesia both in a model of streptozocin-induced diabetic neuropathy and after sciatic nerve ligation without affecting the spontaneous locomotor activity using an actimeter. ${ }^{49} \mathrm{It}$ is therefore possible that the dose tested in the present study was suboptimal, and therefore it cannot be excluded that clomipramine could also be effective in reducing the increased isolated face grooming.

Historically, (neuropathic) pain has been classified on the basis of its etiology (eg, painful diabetic neuropathy, postherpetic neuralgia, trigeminal neuralgia $[\mathrm{TN}]$, and spinal cord injury) and the location of the lesion (peripheral or central). ${ }^{50}$ The growing knowledge of neurobiological mechanisms involved in neuropathic pain has led authors to propose a more mechanism-based approach to pain management. ${ }^{51-53}$ Different somatosensory symptoms of neuropathic pain have been linked to different mechanisms and may have specific pharmacological characteristics. Vos et $\mathrm{al}^{7}$ reported that the behavioral signs of the two different classes of neuropathic sensory dysfunctions in the IoN-CCI model follow a different time course and suggested that this may indicate that different neuropathological mechanisms underlie these symptoms. In keeping with this, we found that the anticonvulsant, carbamazepine, the GABA-B agonist, baclofen, the opiate, morphine, and the tricyclic antidepressant, clomipramine, were selectively active against either spontaneous pain or mechanical hypersensitivity following IoN ligation. Furthermore, a significant decrease in the isolated face grooming was found in rats treated with carbamazepine and baclofen 2 weeks after pumps were removed, whereas no significant effect was found on the development of mechanical allodynia 1 day earlier. These data indicate that different mechanisms may be involved in these separate classes of behavioral changes and thus respond differentially to different treatments.

\section{Conclusion}

The results obtained with carbamazepine and baclofen provide pharmacological support for the use of face grooming as behavioral measure of spontaneous neuropathic pain and further validates the IoN-CCI model for the study of (trigeminal) neuropathic pain in general and TN in particular. Differential drug effects on spontaneous and touch-evoked signs of neuropathic pain indicate that different neuropathological mechanisms may underlie these different somatosensory symptoms in the IoNCCI model. A mechanism-based approach may therefore be needed to more effectively treat (trigeminal) neuropathic pain.

\section{Acknowledgment}

The authors thank K. Szewczyk for assistance in conducting the experiments.

\section{Disclosure}

The authors report no conflicts of interest in this work.

\section{References}

1. Orza F, Boswell MV, Rosenberg SK. Neuropathic pain: review of mechanisms and pharmacologic management. NeuroRehabilitation. 2000;14(1):15-23.

2. Abbott FV, Franklin KB, Westbrook RF. The formalin test: scoring properties of the first and second phases of the pain response in rats. Pain. 1995;60(1):91-102.

3. Colpaert FC. Evidence that adjuvant arthritis in the rat is associated with chronic pain. Pain. 1987;28(2):201-222.

4. Colpaert FC, Tarayre JP, Alliaga M, Bruins Slot LA, Attal N, Koek W. Opiate self-administration as a measure of chronic nociceptive pain in arthritic rats. Pain. 2001;91(1-2):33-45.

5. Mogil JS, Crager SE. What should we be measuring in behavioral studies of chronic pain in animals? Pain. 2004;112:12-15.

6. Gottrup H, Bach FW, Juhl G, Jensen TS. Differential effect of ketamine and lidocaine on spontaneous and mechanical evoked pain in patients with nerve injury pain. Anesthesiology. 2006;104(3):527-536.

7. Vos BP, Strassman AM, Maciewicz RJ. Behavioral evidence of trigeminal neuropathic pain following chronic constriction injury to the rat's infraorbital nerve. J Neurosci. 1994;14(5 pt 1):2708-2723.

8. Deseure K, Adriaensen H. Nonevoked facial pain in rats following infraorbital nerve injury: a parametric analysis. Physiol Behav. 2004;81(4):595-604.

9. Deseure K, Hans G. Behavioral study of non-evoked orofacial pain following different types of infraorbital nerve injury in rats. Physiol Behav. 2015;138:292-296.

10. McQuay H, Carroll D, Jadad AR, Wiffen P, Moore A. Anticonvulsant drugs for management of pain: a systematic review. BMJ. 1995;311(7012): 1047-1052.

11. Fields HL. Treatment of trigeminal neuralgia. N Engl J Med. 1996; 334(17):1125-1126.

12. Backonja MM. Use of anticonvulsants for treatment of neuropathic pain. Neurology. 2002;59(5 suppl 2):S14-S17.

13. Idänpään-Heikkilä JJ, Guilbaud G. Pharmacological studies on a rat model of trigeminal neuropathic pain: baclofen, but not carbamazepine, morphine or tricyclic antidepressants, attenuates the allodynia-like behaviour. Pain. 1999;79(2-3):281-290.

14. Fromm GH, Terrence CF, Chattha AS. Baclofen in the treatment of trigeminal neuralgia: double-blind study and long term follow up. Ann Neurol. 1984;15(3):240-244.

15. Attal N. Pharmacologic treatment of neuropathic pain. Acta Neurol Belg. 2001;101(1):53-64.

16. Sindrup SH, Jensen TS. Pharmacotherapy of trigeminal neuralgia. Clin J Pain. 2002;18(1):22-27. 
17. Deseure KR, Adriaensen HF. Comparison between two types of behavioral variables of non-evoked facial pain after chronic constriction injury to the rat infraorbital nerve. Comp Med. 2002;52(1):44-49.

18. Deseure K, Koek W, Adriaensen H, Colpaert FC. Continuous administration of the 5-hydroxytryptamine1A agonist (3-Chloro-4-fluorophenyl)-[4-fluoro-4-[[(5-methyl-pyridin-2-ylmethyl)-amino]-methyl] piperidin-1-yl]-methadone (F 13640) attenuates allodynia-like behavior in a rat model of trigeminal neuropathic pain. $J$ Pharmacol Exp Ther. 2003;306(2):505-514.

19. Zimmerman M. Ethical Guidelines for investigations of experimental pain in conscious animals. Pain. 1983;16(2):109-110.

20. Gregg JM. A surgical approach to the ophthalmic-maxillary nerve trunks in the rat. $J$ Dent Res. 1973;52(2):392.

21. Jacquin MF, Zeigler HP. Trigeminal orosensation and ingestive behavior in the rat. Behav Neurosci. 1983;97(1):62-97.

22. Bennet GJ, Xie YK. A peripheral mononeuropathy in rat that produces disorders of pain sensation like those seen in man. Pain. 1988; 33(1):87-107.

23. Cain SM, Ruest T, Pimlott $\mathrm{S}$, et al. High resolution micro-SPECT scanning in rats using $125 \mathrm{I}$ beta-CIT: effects of chronic treatment with carbamazepine. Epilepsia. 2009;50(8):1962-1970.

24. Doheny HC, Whittington MA, Jefferys JG, Patsalos PN. A comparison of the efficacy of carbamazepine and the novel anti-epileptic drug levetiracetam in the tetanus toxin model of focal complex partial epilepsy. Br J Pharmacol. 2002;135(6):1425-1434.

25. Deseure K, Bréand S, Colpaert FC. Curative-like analgesia in a neuropathic pain model: parametric analysis of the dose and the duration of treatment with a high-efficacy 5-HT(1A) receptor agonist. Eur $J$ Pharmacol. 2007;568(1-3):134-141.

26. Sindrup SH, Jensen TS. Efficacy of pharmacological treatments of neuropathic pain: an update and effect related to mechanism of drug action. Pain. 1999;83(3):389-400.

27. Sindrup SH, Jensen TS. Pharmacologic treatment of pain in polyneuropathy. Neurology. 2000;55(7):915-920.

28. Collins SL, Moore RA, McQuay HJ, Wiffen P. Antidepressants and anticonvulsants for diabetic neuropathy and postherpetic neuralgia: a quantitative systematic review. J Pain Symptom Manage. 2000;20(6):449-458.

29. Dray A. Agonists and antagonists of nociception. In: Jensen TS, Turner JA, Wiesenfeld-Hallin Z, editors. Proceedings of the 8th World Congress on Pain. Seattle: IASP Press; 1997:279-292.

30. Burchiel KJ. Carbamazepine inhibits spontaneous activity in experimental neuromas. Exp Neurol. 1988;102(2):249-253.

31. Devor M, Govrin-Lippmann R, Angelides K. Na+ channel immunolocalization in peripheral mammalian axons and changes following nerve injury and neuroma formation. J Neurosci. 1993;13(5):1976-1992.

32. England JD, Gamboni F, Ferguson MA, Levinson SR. Sodium channels accumulate at the tips of injured axons. Muscle Nerve. 1994;17(6): 593-598.

33. Matzner O, Devor M. Hyperexcitability at sites of nerve injury depends on voltage-sensitive Na+ channels. J Neurophysiol. 1994;72(1):349-359.

34. Han HC, Lee DH, Chung JM. Characteristics of ectopic discharges in a rat neuropathic pain model. Pain. 2000;84(2-3):253-261.

35. Campbell JN. Nerve lesions and the generation of pain. Muscle Nerve. 2001;24(10):1261-1273.
36. De Vry J, Kuhl E, Franken-Kunkel P, Eckel G. Pharmacological characterization of the chronic constriction injury model of neuropathic pain. Eur J Pharmacol. 2004;491(2-3):137-148.

37. Foong FW, Satoh M. Neurotransmitter-blocking agents influence antinociceptive effects of carbamazepine, baclofen, pentazocine and morphine on bradykinin-induced trigeminal pain. Neuropharmacology. 1984; 23(6):633-636.

38. Arner S, Meyerson BA. Lack of analgesic effect of opioids on neuropathic and idiopathic forms of pain. Pain. 1988;33(1):11-23.

39. Portenoy RK, Foley KM, Inturrisi CE. The nature of opioid responsiveness and its implications for neuropathic pain: new hypotheses derived from studies of opioid infusions. Pain. 1990;43(3):273-286.

40. Bulka A, Plesan A, Xu XJ, Wiesenfeld-Hallin Z. Reduced tolerance to the anti-hyperalgesic effect of methadone in comparison to morphine in a rat model of mononeuropathy. Pain. 2002;95(1-2):103-109.

41. Yu W, Hao JX, Xu XJ, Wiesenfeld-Hallin Z. The development of morphine tolerance and dependence in rats with chronic pain. Brain Res. 1997;756(1-2):141-146.

42. DeMulder PA, Claus M, DeMulder G, Adriaensen H, Vos BP. Effects of morphine and ketamine on behavioral signs of increased trigeminal nociceptive activity after chronic constriction injury to the rat's infraorbital nerve. Soc Neurosci Abstr. 1994;20:555.

43. Deseure K, Koek W, Colpaert FC, Adriaensen H. The 5-HT(1A) receptor agonist $\mathrm{F} 13640$ attenuates mechanical allodynia in a rat model of trigeminal neuropathic pain. Eur J Pharmacol. 2002;456(1-3):51-57.

44. Bromage PR, Camporesi EM, Durant PA, Nielsen CH. Nonrespiratory side effects of epidural morphine. Anesth Analg. 1982;61(6): 490-495.

45. Moser HR, Giesler GJ Jr. Itch elicited by intradermal injection of serotonin, intracisternal injection of morphine, and their synergistic interactions in rats. Neuroscience. 2014;274:119-127.

46. Klein A, Carstens MI, Carstens E. Facial injections of pruritogens or algogens elicit distinct behavior responses in rats and excite overlapping populations of primary sensory and trigeminal subnucleus caudalis neurons. J Neurophysiol. 2011;106(3):1078-1088.

47. Spradley JM, Davoodi A, Carstens MI, Carstens E. Opioid modulation of facial itch- and pain-related responses and grooming behavior in rats. Acta Derm Venereol. 2012;92(5):515-520.

48. Ardid D, Guilbaud G. Antinociceptive effects of acute and 'chronic' injections of tricyclic antidepressant drugs in a new model of mononeuropathy in rats. Pain. 1992;49(2):279-287.

49. Wattiez AS, Libert F, Privat AM, et al. Evidence for a differential opioidergic involvement in the analgesic effect of antidepressants: prediction for efficacy in animal models of neuropathic pain? Br J Pharmacol. 2011;163(4):792-803.

50. Merskey H, Bogduk N. Classification of Chronic Pain, Second Edition, IASP Task Force on Taxonomy. Seattle: IASP Press; 1994.

51. Jensen TS. An improved understanding of neuropathic pain. Eur J Pain. 2002;6(Suppl B):3-11.

52. Woolf CJ. Dissecting out mechanisms responsible for peripheral neuropathic pain: implications for diagnosis and therapy. Life Sci. 2004;74(21):2605-2610.

53. Baron R, Binder A, Wasner G. Neuropathic pain: diagnosis, pathophysiological mechanisms, and treatment. Lancet Neurol. 2010;9(8):807-881.
Journal of Pain Research

\section{Publish your work in this journal}

The Journal of Pain Research is an international, peer reviewed, open access, online journal that welcomes laboratory and clinical findings in the fields of pain research and the prevention and management of pain. Original research, reviews, symposium reports, hypothesis formation and commentaries are all considered for publication.

\section{Dovepress}

The manuscript management system is completely online and includes a very quick and fair peer-review system, which is all easy to use. Visit http://www.dovepress.com/testimonials.php to read real quotes from published authors. 